\title{
Influence of Sleep Deprivation on Neuroactive Steroids in Major Depression
}

\author{
Cornelius Schüle', Flavia di Michele², Thomas Baghai', Elena Romeo'2, Giorgio Bernardi², Peter Zwanzger', \\ Frank Padberg', Augusto Pasini ${ }^{2}$ and Rainer Rupprecht*, I,3 \\ 'Department of Psychiatry, Ludwig-Maximilian-University, Munich, Germany; ${ }^{2}$ RCCS Santa Lucia, Tor Vergata University, Rome, Italy; ${ }^{3}$ Max \\ Planck Institute of Psychiatry, Munich, Germany
}

\begin{abstract}
There is evidence from preclinical and clinical studies that concentrations of neuroactive steroids are altered in depression and normalize after antidepressant pharmacotherapy. However, no data are available concerning the impact of sleep deprivation on the concentrations of neuroactive steroids. A total of 29 drug-free patients ( 12 men, 17 women) suffering from major depression according to DSM-IV criteria were treated with partial sleep deprivation (PSD). Response to PSD was defined as a reduction of at least 30\% according to the six-item version of the Hamilton depression scale (6-HAMD). Plasma samples were taken the day before and after PSD (days 0 and I) and after one night of recovery sleep (day 2) at 8:00 am. The samples were quantified for neuroactive steroids by means of a highly sensitive and specific combined gas chromatography/mass spectrometry analysis. There was no influence of PSD on the concentrations of neuroactive steroids either in PSD responders $(n=20)$ or in nonresponders $(n=9)$. However, nonresponders showed significantly higher concentrations of $3 \alpha, 5 \alpha$-tetrahydroprogesterone ( $3 \alpha, 5 \alpha$-THP), $3 \alpha, 5 \beta$-tetrahydroprogesterone $(3 \alpha, 5 \beta$-THP), and dehydroepiandrosterone (DHEA) before or after PSD compared to responders. In contrast to antidepressant drugs, which correct the dysequilibrium of neuroactive steroids in major depression within several weeks, PSD does not affect the concentrations of neuroactive steroids either in responders or in nonresponders.

Neuropsychopharmacology (2003) 28, 577-58I. doi:I0.1038/s..npp. 1300084
\end{abstract}

Keywords: neurosteroids; neuroactive steroids; progesterone; sleep deprivation; major depression; depressive disorder

\section{INTRODUCTION}

Certain $3 \alpha$-reduced metabolites of progesterone such as $3 \alpha, 5 \alpha$-tetrahydroprogesterone ( $3 \alpha, 5 \alpha$-THP, $5 \alpha$-pregnan- $3 \alpha$ ol-20-one, allopregnanolone) and $3 \alpha, 5 \beta$-tetrahydro-progesterone $(3 \alpha, 5 \beta$-THP, $5 \beta$-pregnan- $3 \alpha$-ol-20-one, pregnanolone) are potent positive allosteric modulators of the $\gamma$-aminobutyric $\operatorname{acid}_{\mathrm{A}}\left(\mathrm{GABA}_{\mathrm{A}}\right)$ receptor complex and may regulate gene expression via the progesterone receptor after intracellular oxidation (Paul and Purdy, 1992; Rupprecht and Holsboer, 1999). It has been shown that selective serotonin reuptake inhibitors (SSRIs) may enhance the formation of $3 \alpha$-reduced neuroactive steroids both in experimental animals (Uzunov et al, 1996) and at the molecular level (Griffin and Mellon, 1999). Moreover, there is evidence that such steroids might contribute to the pathophysiology of depression and its treatment by antidepressant drugs. During depression, the concentra-

\footnotetext{
*Correspondence: Dr R Rupprecht, Department of Psychiatry, Ludwig-Maximilian-University, Nussbaumstr. 7, 80336 Munich, Germany, Tel: +49 895160 2770, Fax: +49 895160 5524, E-mail: Rainer.Rupprecht@psy.med.uni-muenchen.de

Received 7 May 2002; revised 26 August 2002; accepted 2 October 2002

Online publication: 10 October 2002 at http://www.acnp.org/citations/ Npp I01002403
}

tions of $3 \alpha, 5 \alpha$-THP and $3 \alpha, 5 \beta$-THP were decreased, while the levels of $3 \beta, 5 \alpha$-tetrahydroprogesterone ( $3 \beta, 5 \alpha$-THP, $5 \alpha-$ pregnan-3 $\beta$-ol-20-one, isopregnanolone), a stereoisomer of $3 \alpha, 5 \alpha$-THP, which may act as an antagonist for GABAergic steroids (Rupprecht and Holsboer, 1999), were increased (Romeo et al, 1998). Treatment with both SSRIs (Romeo et al, 1998; Uzunova et al, 1998) and tricyclic antidepressants (Romeo et al, 1998) increases $3 \alpha$-reduced pregnane steroids and decreases $3 \beta, 5 \alpha$-THP (Romeo et al, 1998) for several weeks. Furthermore, administration of dehydroepiandrosterone (DHEA, 5 -androsten-3 $\beta$-ol-17-one) has been shown to alleviate depressive symptoms in a placebo-controlled study (Wolkowitz et al, 1999).

Currently, it is not known whether changes in the concentrations of neuroactive steroids are a general principle of clinically effective antidepressant treatment or whether they are related to direct pharmacological effects of antidepressant drugs. Sleep deprivation rapidly alleviates depressive symptoms in about $60 \%$ of patients with major depressive disorder ( $\mathrm{Wu}$ and Bunney, 1990; Wirz-Justice and Van den Hoofdakker, 1999). However, the positive effects of sleep deprivation on mood are only transient as usually there is a clinical deterioration after one night of recovery sleep. In order to investigate whether mood changes are accompanied by changes in neuroactive steroids, we studied the impact of partial sleep deprivation 
(PSD) as a nonpharmacological treatment intervention on plasma levels of progesterone, $3 \alpha, 5 \alpha$-THP, $3 \alpha, 5 \beta$-THP, $3 \beta, 5 \alpha$-THP, and DHEA in depressed patients and compared responders with nonresponders.

\section{METHODS}

\section{Subjects}

A total of 29 drug-free depressed inpatients (12 men, 17 women) aged between 27 and 77 years (mean $=53.66$, $\mathrm{SD}=11.91$ ) entered the study after the procedures had been fully explained and written informed consent had been obtained. The study was conducted in accordance with the Declaration of Helsinki. Inclusion criteria were: (a) a major depressive episode according to DSM-IV criteria, (b) a score of at least 18 on the 21-item Hamilton depression rating scale (21-HAMD), (c) exclusion of major medical disorders, addiction, or other comorbid psychiatric diagnoses, (d) no psychotropic drugs for at least 5 days before and throughout the study and (e) exclusion of pregnancy or use of oral contraceptives. None of the patients had been pretreated with fluoxetine. On the day of PSD, patients went to bed between 9:00 and 11:00 pm and were awakened at 1.30 am. They spent the rest of the night in a social room of the ward and were supervised by a nurse. On the following day, particular care was taken to prevent patients from sleeping or taking a nap before their regular bedtime. The PSD was followed by a night during which the patients were allowed to sleep according to their usual habits.

Severity of depression was estimated with a 21-item version and a 6-item version of the Hamilton Depression Rating Scale (21-HAMD, 6-HAMD). The 6-HAMD is a psychometric instrument suitable for repeated measurements and detection of rapid mood changes (Bech et al, 1975), which covers depressed mood, guilt feelings, work and interest, psychomotor retardation, anxiety (psychic), and physical symptoms. 21-HAMD scores were collected at baseline (day 0) and 6-HAMD scores were evaluated every day between 11:00 and 12:00 am prior to PSD (day 0), the day after PSD (day 1), and 2 days after PSD (day 2). Response to PSD was defined as a reduction of at least $30 \%$ in the 6HAMD score between days 0 and 1 (Wiegand et al, 1993).

\section{Quantification of Neuroactive Steroids and Cortisol}

Plasma samples were taken at 8:00 am the day before PSD (day 0 ) and 2 days thereafter (days 1 and 2). They were quantified for neuroactive steroids (progesterone, $3 \alpha, 5 \alpha$ THP, $3 \alpha, 5 \beta$-THP, $3 \beta, 5 \alpha$-THP, DHEA) by means of a highly sensitive and specific combined gas chromatography/mass spectrometry analysis (GC/MS) after extraction with ethyl acetate (Strohle et al, 2002). A Finningham Trace GC/MS equipped with a capillary column was used to analyze the derivatized steroids in the negative ion chemical ionization mode. The detection limit was approximately $10 \mathrm{fmol}$. Moreover, plasma cortisol concentrations were determined on day 0 at 8:00 am using a commercial radioimmunoassay kit (Cortisol RIA, DPC Biermann, Germany) to calculate the cortisol/DHEA ratio. The lower detection limit of this cortisol RIA was $8.27 \mathrm{nmol} / \mathrm{l}$ with intra- and interassay coefficients of variation below $5 \%$.

\section{Statistical Analyis}

Homogeneity between PSD responders and nonresponders in some demographic variables was analyzed by the $\chi^{2}$ test for contingency tables or Fisher's exact test with respect to qualitative variables (gender; type of premedication subdivided into tricyclics, SSRIs, other antidepressants or benzodiazepines) or by two-tailed $t$-tests for independent samples with regard to quantitative variables (age; length of the drug-free interval; 21-HAMD and 6-HAMD sum scores). Moreover, plasma levels of cortisol and cortisol/DHEA ratios on day 0 were compared between responders and nonresponders by means of the two-tailed $t$-test for independent samples. Correlations between levels of neuroactive steroids, age and both severity of depression (21HAMD sum scores) at baseline and clinical improvement after PSD (percental change in 6-HAMD sum scores) were tested about significance using Pearson's correlation coefficient. For statistical comparisons of mean steroid concentrations before (day 0) and after PSD (days 1 and 2), and between responders and nonresponders multivariate analysis of covariance (MANCOVA) with a repeatedmeasures design was performed. Thereby 'treatment' (PSD) and 'response' were considered as within-subjects and between-subjects factors with three and two levels, respectively, and 'age' and 'gender' as covariates. MANCOVA with a repeated-measures design was also carried out to test the effects of 'treatment' and 'gender' on the steroids without differentiating between responders and nonresponders. Here, only 'age' was considered as a covariate. When a significant factor effect was found in the MANCOVAs, univariate F-tests followed to identify those neuroactive steroids that contributed significantly to these effects. For these steroids, tests with contrasts were additionally performed when treatment was among the significant influential factors. As a nominal level of significance, $\alpha=0.05$ was accepted. To keep the type I error equal to 0.05 , post hoc univariate F-tests were performed at a reduced level of significance ( $\alpha$ adjusted according to the Bonferroni procedure).

\section{RESULTS}

A total of $69.0 \%$ of depressed patients (20 out of 29) responded to PSD. The therapeutic effect of PSD was only transient in that there was a deterioration of depressive symptoms on day 2 in the responder group (Table 1). Responders $(n=20)$ and nonresponders $(n=9)$ did not differ significantly in gender distribution (Fisher's exact test), type of psychopharmacological pretreatment ( $\chi^{2}$ test), length of the drug-free interval, 6-HAMD or 21-HAMD sum scores ( $t$-test for independent samples; Table 1$)$. Responders ( 8 men, 12 women) had been pretreated with tricyclic antidepressants $(n=6)$, SSRIs $(n=5)$, other antidepressants $(n=4)$, or benzodiazepines $(n=5)$ before entering the study, whereas nonresponders ( 4 men, 5 women) had received tricyclic antidepressants $(n=3)$, SSRIs $(n=3)$, other antidepressants $(n=1)$, or benzodiazepines $(n=2)$ before the washout period. Since there was a significant difference in the mean age between PSD responders $(50.65 \pm 11.40$ years $)$ and nonresponders $(60.33 \pm 10.71$ 
Table I Clinical and Endocrinological Data in Responders $(n=20)$ and Nonresponders $(n=9)$ to PSD

\begin{tabular}{|c|c|c|c|c|c|c|c|}
\hline & \multirow{2}{*}{$\begin{array}{l}\text { Drug-free } \\
\text { interval (days) }\end{array}$} & \multirow{2}{*}{$\begin{array}{l}\text { Cortisol } \\
(\mathrm{nmol} / \mathrm{l})\end{array}$} & \multirow{2}{*}{$\begin{array}{l}\text { Cortisol/DHEA } \\
\text { ratio }\end{array}$} & \multirow{2}{*}{$\begin{array}{l}\text { 2I-HAMD } \\
\text { Day } 0\end{array}$} & \multicolumn{3}{|l|}{ 6-HAMD } \\
\hline & & & & & Day 0 & Day I & Day 2 \\
\hline Responders $(n=20)$ & $8.20(6.44)$ & $473.8(177.8)$ & $30.70(19.38)$ & $26.95(6.09)$ & I2.35 (2.89) & $4.10(2.22)$ & $8.90(4.70)$ \\
\hline Nonresponders $(n=9)$ & $8.56(9.93)$ & $408.0(\mid$ | 8.4$)$ & $19.94(21.98)$ & $24.44(6.48)$ & $10.67(3.61)$ & $10.11(3.44)$ & $9.89(3.52)$ \\
\hline
\end{tabular}

Data represent the mean $( \pm \mathrm{SD})$.
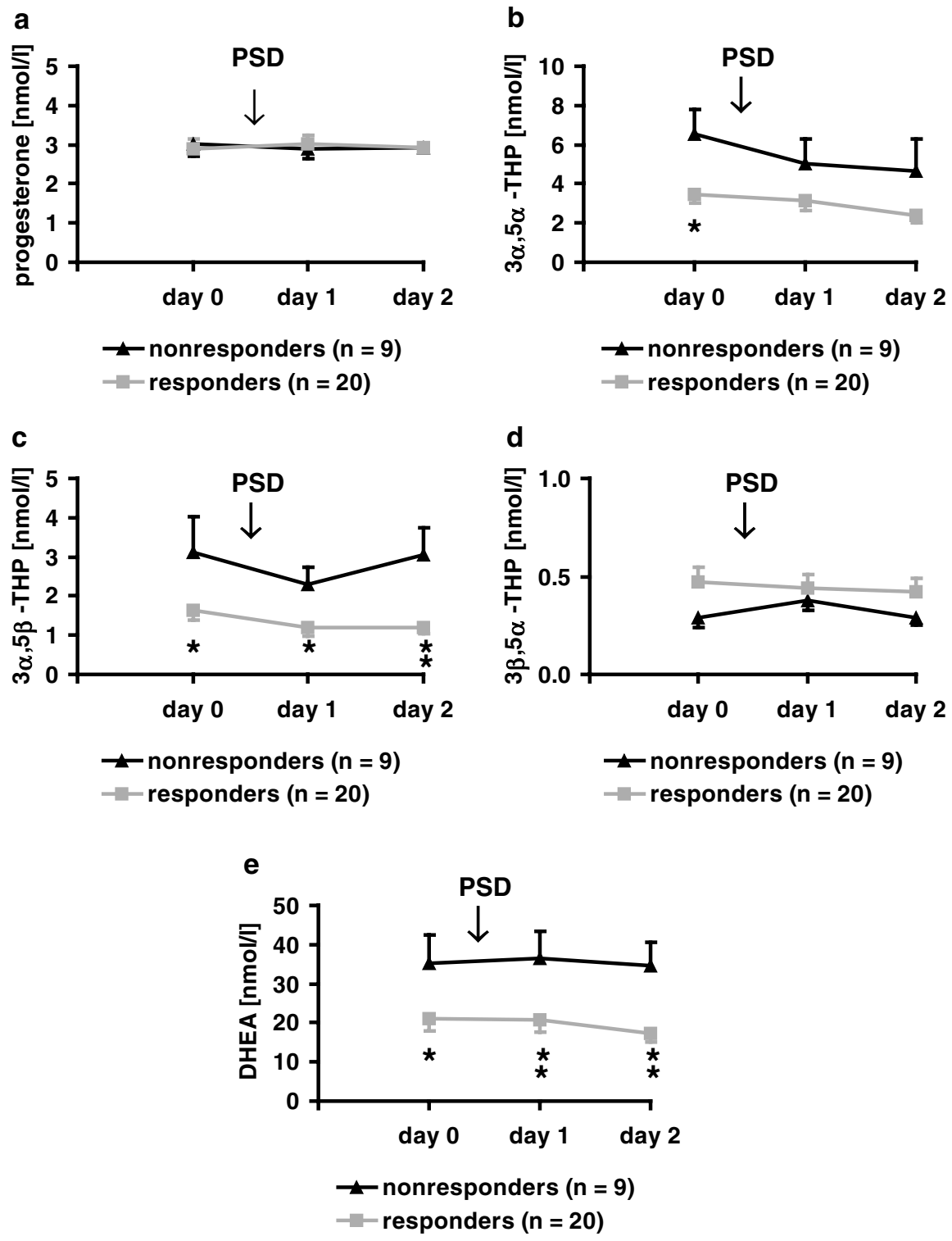

Figure I Concentrations of progesterone (a), $3 \alpha, 5 \alpha-T H P(b), 3 \alpha, 5 \beta$-THP (c), $3 \beta, 5 \alpha$-THP (d), and DHEA (e) in nonresponders and responders to PSD on day 0 up to day 2 . Data represent the mean $( \pm$ SEM). *Statistical significance at the $p<0.05$ level in tests with contrast following MANCOVA. **Statistical significance at the $p<0.01$ level in tests with contrasts following MANCOVA.

years), 'age' was used as a covariate in the analysis of variance.

Both plasma cortisol levels and cortisol/DHEA ratios did not differ significantly between responders and nonresponders on day 0 ( $t$-test for independent samples; Table 1). At baseline and the subsequent days, the concentrations of neuroactive steroids were comparable between men and women (MANCOVA, Wilks' multivariate tests of significance; effect of 'gender': $F_{(5,22)}=1.31$; significance of $\mathrm{F}=0.297$, effect of 'gender by treatment': $\mathrm{F}_{(10,18)}=1.19$, significance of $\mathrm{F}=0.357$ ). By considering 'treatment' and 'response' as influential factors and 'gender' and 'age' as covariates, analysis of covariance did not reveal any significant effect of 'treatment', nor of 'treatment by 
response' interaction on the concentrations of neuroactive steroids (Figure 1). However, MANCOVA indicated a significant main effect of 'response' (Wilks' multivariate tests of significance; effect of 'treatment': $F_{(10,18)}=1.70$, significance of $\mathrm{F}=0.157$; effect of 'treatment by response': $\mathrm{F}_{(10,18)}=0.87$, significance of $\mathrm{F}=0.577$; effect of 'response': $\mathrm{F}_{(5,21)}=6.76$, significance of $\left.\mathrm{F}=0.001\right)$. This 'response' effect was attributed mainly to $3 \alpha, 5 \beta$-THP and DHEA levels and to $3 \alpha, 5 \alpha$-THP concentrations (univariate F-tests with $\mathrm{df}=1,25 ; p=0.009,0.001$, and 0.053 , respectively). For $3 \alpha, 5 \beta$-THP and DHEA, tests with contrasts pointed out significant differences between responders and nonresponders to PSD on day 0 up to day $2(p<0.05)$. Compared to responders, nonresponders showed significantly higher levels in $3 \alpha, 5 \beta$-THP and DHEA throughout the time period. Similarly, nonresponders were prone to have higher concentrations of $3 \alpha, 5 \alpha$-THP than responders on all days, but there was only a significant difference on day 0 (tests with contrasts, $p<0.05)$. Progesterone and $3 \beta, 5 \alpha$-THP concentrations did not differ between nonresponders and responders to PSD (Figure 1).

No significant correlation between age and baseline DHEA levels could be demonstrated in our study
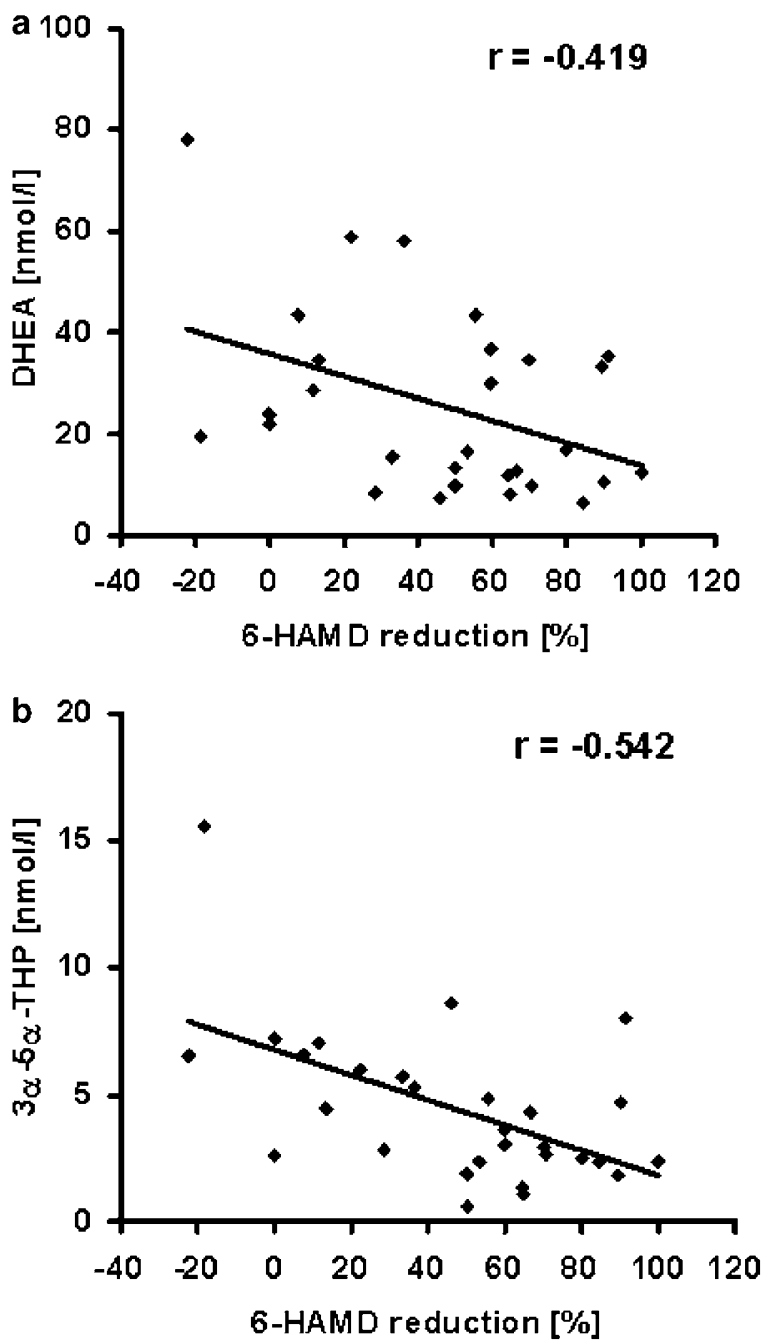

Figure 2 Scatterplot of the percental 6-HAMD reduction after one night of PSD and DHEA (a) or $3 \alpha-5 \alpha$-THP (b) concentrations in 29 depressive patients. $(r=-0.192)$. There was neither a significant association between levels of neuroactive steroids and 21-HAMD sum scores at baseline nor between percental change in neuroactive steroids and percental alteration of 6-HAMD sum scores between days 0 and 1 ( $r$ between -0.239 and 0.092 ). However, significant negative correlations could be demonstrated between percental 6-HAMD reduction (days 0 and 1) and baseline concentrations of DHEA $(r=-0.419$; $p=0.024)$ and $3 \alpha, 5 \alpha$-THP $(r=-0.542 ; p=0.002)$, that is, higher DHEA and $3 \alpha, 5 \alpha$-THP levels at baseline were associated with lower clinical improvement after PSD (Figure 2). No association with responsiveness to PSD was found with respect to $3 \alpha, 5 \beta$-THP, $3 \beta, 5 \alpha$-THP, and progesterone concentrations. Moreover, cortisol levels and cortisol/DHEA ratios at baseline did not correlate significantly with percental changes in 6-HAMD sum scores ( $r$ between 0.033 and 0.265 ).

\section{DISCUSSION}

Since circadian fluctuations in the concentrations of progesterone and its metabolites have been demonstrated in an animal study (Corpechot et al, 1997), neuroactive steroids were quantified at the same time point $(8: 00 \mathrm{am})$ on each study day in our investigation. Apparently, the elevated concentrations of certain neuroactive steroids $(3 \alpha, 5 \alpha$-THP, $3 \alpha, 5 \beta$-THP, DHEA) in PSD nonresponders compared to responders were not related to gender, type of psychopharmacological pretreatment, or length of the drugfree interval because both groups were comparable in these parameters. Although DHEA levels decline with age (Baulieu, 1996) and nonresponders to PSD were older than responders in our investigation, the elevated DHEA concentrations in nonresponders to PSD were scarcely determined by age as shown by the analysis of covariance. Moreover, gender did not affect our results since the gender distribution was comparable in responders and nonresponders to PSD.

The elevated levels of the respective neuroactive steroids in PSD nonresponders and the negative correlation between baseline levels of two steroids (DHEA, $3 \alpha, 5 \alpha$-THP) and clinical responsiveness to PSD may reflect the hyperactivity of the hypothalamic-pituitary-adrenal system in nonresponders to sleep deprivation (Schule et al, 2001). In depressive patients, a diurnal hypersecretion of both cortisol and DHEA has been found during pulsatile 24-h sampling (Heuser et al, 1998). Interestingly, increased concentrations of DHEA sulfate have recently been shown to predict also nonresponse to electroconvulsive therapy (Maayan et al, 2000), which is in line with our results. Our observation that baseline cortisol levels did not differ between responders and nonresponders to PSD may be due to the fact that single-point measurements of basal cortisol secretion are often insufficient and intensive monitoring of HPA axis hormones over time is needed to detect elevated cortisol levels in depression (Posener et al, 2000). Since DHEA is a steroid with antiglucocorticoid properties (Kalimi et al, 1994), an enhanced cortisol/DHEA ratio has recently been suggested to be a more precise method to assess the 'functional' hypercortisolemia in depression (Young et al, 2002). In our investigation, the plasma 
cortisol/DHEA ratio at baseline was not associated with the responsiveness to PSD.

An important finding of the present study is that PSD does not influence the concentrations of neuroactive steroids either in responders or in nonresponders to this nonpharmacological treatment. This is in contrast to findings with antidepressants, where response to drug treatment has been demonstrated to be associated with an increase in the levels of GABAergic steroids for several weeks both in the plasma (Romeo et al, 1998) and the cerebrospinal fluid (Uzunova et al, 1998) of depressed patients. In particular, SSRIs but not tricyclic antidepressants may shift the activity of the $3 \alpha$-hydroxysteroid oxidoreductase towards the reductive direction, thereby enhancing the concentrations of $3 \alpha$-reduced neuroactive steroids (Griffin and Mellon, 1999). However, it remains unclear as to whether the effects of antidepressant drugs on neuroactive steroids in depressed patients are related to direct pharmacological actions or due to general clinical improvement and antidepressant efficacy after several weeks of treatment. Apparently, the fast occurring and pronounced alleviation of depressive symptoms in responders to PSD and subsequent clinical deterioration after one night of recovery sleep is not accompanied by changes in the concentrations of neuroactive steroids. Moreover, plasma levels of neuroactive steroids are not influenced by treatment with repetitive transcranial magnetic stimulation for 2 weeks (Padberg et al, 2002). Further studies should investigate the effects of medium-term nonpharmacological antidepressant treatment strategies on neuroactive steroids such as prolonged transcranial magnetic stimulation, electroconvulsive therapy, or vagus nerve stimulation.

\section{ACKNOWLEDGMENTS}

We thank Ms Angela Johnson and Ms Beatrix Geissler for expert technical assistance and Dr Alexander Yassouridis, Max Planck Institute of Psychiatry, Munich, Germany, for the skillful statistical analysis. This study was supported by the German Ministry for Education and Research within the promotional emphasis 'German Research Network on Depression', subproject 4.3, the Gerhard Hess Programm of the 'Deutsche Forschungsgemeinschaft' (DFG), and the Max Planck Society.

\section{REFERENCES}

Baulieu EE (1996). Dehydroepiandrosterone (DHEA): a fountain of youth? J Clin Endocrinol Metab 81: 3147-3151.

Bech P, Gram LF, Dein E, Jacobsen O, Vitger J, Bolwig TG (1975). Quantitative rating of depressive states. Acta Psychiatr Scand 51: $161-170$

Corpechot C, Collins BE, Carey MP, Tsouros A, Robel P, Fry JP (1997). Brain neurosteroids during the mouse oestrous cycle. Brain Res 766: 276-80.

Griffin LD, Mellon SH (1999). Selective serotonin reuptake inhibitors directly alter activity of neurosteroidogenic enzymes. Proc Natl Acad Sci USA 96: 13512-1357.
Heuser I, Deuschle M, Luppa P, Schweiger U, Standhardt H, Weber B (1998). Increased diurnal plasma concentrations of dehydroepiandrosterone in depressed patients. J Clin Endocrinol Metab 83: 3130-3133.

Kalimi M, Shafagoj Y, Loria R, Padgett D, Regelson W (1994). Antiglucocorticoid effects of dehydroepiandrosterone (DHEA). $\mathrm{Mol}$ Cell Biochem 131: 99-104.

Maayan R, Yagorowski Y, Grupper D, Weiss M, Shtaif B, Kaoud MA et al (2000). Basal plasma dehydroepiandrosterone sulfate level: a possible predictor for response to electroconvulsive therapy in depressed psychotic inpatients. Biol Psychiatry 48: 693-701.

Paul SM, Purdy RH (1992). Neuroactive steroids. FASEB J 6: 23112322.

Padberg F, di Michele F, Zwanzger P, Romeo E, Bernardi G, Schule $C$ et al (2002). Plasma concentrations of neuroactive steroids before and after repetitive transcranial magnetic stimulation (rTMS) in major depression. Neuropsychopharmacology 27: 874878.

Posener JA, DeBattista C, Williams GH, Chmura Kraemer H, Kalehzan BM, Schatzberg AF (2000). 24-Hour monitoring of cortisol and corticotropin secretion in psychotic and nonpsychotic major depression. Arch Gen Psychiatry 57: 755-760.

Romeo E, Strohle A, Spalletta G, di Michele F, Hermann B, Holsboer F et al (1998). Effects of antidepressant treatment on neuroactive steroids in major depression. Am J Psychiatry 155: 910-913.

Rupprecht R, Holsboer F (1999). Neuroactive steroids: mechanisms of action and neuropsychopharmacological perspectives. Trends Neurosci 22: 410-416.

Strohle A, Romeo E, di Michele F, Pasini A, Yassouridis A, Holsboer F et al (2002). GABA(A) receptor-modulating neuroactive steroid composition in patients with panic disorder before and during paroxetine treatment. Am J Psychiatry 159: 145-147.

Schule C, Baghai T, Zwanzger P, Minov C, Padberg F, Rupprecht R (2001). Sleep deprivation and hypothalamic-pituitary-adrenal (HPA) axis activity in depressed patients. J Psychiatr Res 35: 239-247.

Uzunov DP, Cooper TB, Costa E, Guidotti A (1996). Fluoxetineelicited changes in brain neurosteroid content measured by negative ion mass fragmentography. Proc Natl Acad Sci USA 93: 12599-12604

Uzunova V, Sheline Y, Davis JM, Rasmusson A, Uzunov DP, Costa $\mathrm{E}$ et al (1998). Increase in the cerebrospinal fluid content of neurosteroids in patients with unipolar major depression who are receiving fluoxetine or fluvoxamine. Proc Natl Acad Sci USA 95: 3239-3244.

Wirz-Justice A, Van den Hoofdakker RH (1999). Sleep deprivation in depression: what do we know, where do we go? Biol Psychiatry 46: 445-453.

Wu JC, Bunney WE (1990). The biological basis of an antidepressant response to sleep deprivation and relapse: review and hypothesis. Am J Psychiatry 147: 14-21.

Wiegand M, Riemann D, Schreiber W, Lauer CJ, Berger M (1993). Effect of morning and afternoon naps on mood after total sleep deprivation in patients with major depression. Biol Psychiatry 33: $467-476$

Wolkowitz OM, Reus VI, Keebler A, Nelson N, Friedland M, Brizendine L et al (1999). Double-blind treatment of major depression with dehydroepiandrosterone. Am J Psychiatry 156: 646-649.

Young AH, Gallagher P, Porter RJ (2002). Elevation of the cortisolehydroepiandrosterone ratio in drug-free depressed patients. Am J Psychiatry 159: 1237-1239. 